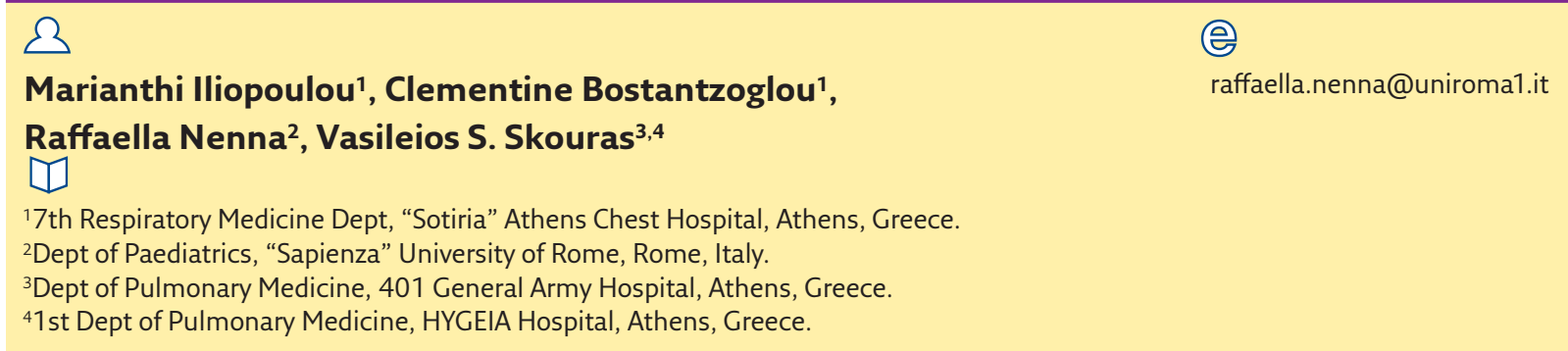

\title{
Asbestos and the lung: highlights of a detrimental relationship
}

\section{Landmark papers in respiratory medicine}

\begin{abstract}
"Asbestos" is a term used to characterise a number of natural mineral fibres of silica that can be categorised according to their structure in the serpentine-type fibres, mainly represented by chrysotile, and the amphibole-type fibres, which include crocidolite, amosite, anthophyllite, actinolite and tremolite [1]. Due to its remarkable durability and fire resistance, asbestos has been used in pottery and clothing since for at least 4500 years. However, modern commercial production of asbestos began during the second half of the 19th century when industry started to exploit its unique chemical characteristics for manufacturing various products, such as pipe insulation, brake linings, cement pipes, protective clothing, etc. With the increasing use of such materials, individuals working in the construction (e.g. builders, plumbers, etc.) and ship building (e.g. dockyard workers) industries began to be exposed to high concentrations of inhaled asbestos fibres. Approximately 50-70 years after the introduction of asbestos in commercial use, the first reports of asbestos-related diseases emerged [1]. Since then, multiple studies have connected asbestos exposure with a variety of malignant and non-malignant lung disorders.
\end{abstract}

\section{Lung cancer}

Asbestos exposure was first suspected as a risk factor for lung cancer in 1935 when the occurrence of this disease was reported in three asbestosis patients [2, 3]. This connection was reinforced by the subsequent report of 61 similar cases, confirmed in 1955, when Doll [4] demonstrated that asbestos workers who had been employed for at least 20 years in places with moderate to high asbestos exposure faced a 10-fold higher risk for lung malignancies compared with the general population. Almost 30 years later, SELIKOFF et al. [5] showed that even light intermittent asbestos exposure was associated with a 6.8-times higher incidence of lung cancer compared with the general population. Indeed, in a large retrospective study of 8580 individuals employed in the asbestos industry during a 60-year period in Denmark, RAFFN et al. demonstrated: first, that the incidence of lung cancer was not dependent on the level of asbestos exposure but only on the duration; and second, that asbestos exposure was associated with an increase in the incidence of only adenocarcinoma [6]. Although several researchers agree that asbestos exposure is accompanied by increased lung
Cite as: Iliopoulou M, Bostantzoglou C, Nenna R, et al. Asbestos and the lung: highlights of a detrimental relationship. Breathe 2017; 13: 235-237. 
adenocarcinoma incidence, especially in the lower lung lobes, the contradicting results of other studies, which report higher risk ratios for small cell lung cancer, mean safe conclusions cannot yet be drawn regarding the most prevalent lung cancer histology among asbestos-exposed individuals [7, 8]. Given that both asbestos and tobacco smoking have been associated with increased lung cancer incidence, the question of the level of lung cancer risk in individuals with concurrent exposure to both arose. In a retrospective study of 98912 asbestos workers, Frost et al. [9] demonstrated that the interaction between smoking and asbestos exposure was greater than additive (i.e. multiplicative) to the occurrence of lung cancer, while lung cancer risk remained increased even 40 years after smoking cessation.

\section{Mesothelioma}

Apart from a few early reports, the relationship between asbestos exposure and malignant pleural mesothelioma (MPM) was most comprehensively described after the 1950s when an explosion in asbestos use in industrialised countries took place [10-12]. Although an increase in the incidence of MPM was initially seen in mine workers or residents of asbestos mining areas who were exposed to airborne asbestos fibres, subsequent reports suggested that the population at risk might be much larger due to the widespread use of asbestos fibres in a variety of settings, such as insulation and fireproof material in modern buildings and water pipes [10, 12]. Approximately 40 years after the first reliable evidence of an association between asbestos exposure and MPM occurence in 1965, ButNor et al. [13] demonstrated that the employees of 12 industries (shipbuilding, the navy, construction, insulation, oil and chemical industry, on a power plant, on a railroad, automotive industry, steel/metal industry, asbestos manufacturing, at a papermill, ceramic/glassware industry) or those in six possible occupations (pipefitter, boilermaker, maintenance, machinist, electrician, sheetmetal production) were at greatest risk of MPM, along with their household contacts. Interestingly, not all types of asbestos fibre have been associated with MPM. The shape of the fibres has been found to play a primary role in carcinogenicity, with fibres $>8 \mu \mathrm{m}$ in length and $<1.5 \mu \mathrm{m}$ in diameter (i.e. the amphibole-type fibres) associated with a higher risk of mesothelioma $[14,15]$. Despite the existence of a dose-damage relationship, no definite asbestos dose has been yet determined as inducing MPM [16]. What has been determined, however, is the potentially long (up to 45 years) incubation period between first asbestos exposure and MPM occurrence, which explains why the incidence of MPM is expected to increase during coming decades despite the ban on asbestos use in many countries across the world $[11,17,18]$.

\section{Benign diseases}

The inhalation of asbestos fibres has also been associated with a number of non-malignant disorders, mainly asbestosis (chronic fibrotic lung disease characterised by diffuse interstitial fibrosis frequently associated with pleural thickening and/ or calcification), benign pleural effusion, pleural plaques (calcified collagenous structures that are almost always bilateral and most commonly located at the parietal pleura of the lower thorax and the diaphragm), pleural thickening and rounded atelectasis. Although the first cases of asbestosis were reported at the beginning of the 20th century, Cooke and McDonald were those who provided a comprehensive description of the clinical, radiological and histological presentation of asbestosis in asbestos-exposed individuals in 1927 [19-21]. Subsequent studies in 1960-1970 showed that modest asbestos exposure (i.e. relatives of asbestos workers or individuals living near asbestos factories) to high asbestos exposure (i.e. asbestos workers) can also affect the pleura through the development of pleural plaques [22]. Recently, PARIs et al. [23] demonstrated that the development of pleural plaques depends on both the duration and level (i.e. cumulative dose of inhaled asbestos fibres, with "high level" defined as continuous exposure for $\geq 1$ year or discontinuous exposure for $\geq 10$ years and "low level" defined as passive exposure) of asbestos exposure while the occurrence of asbestosis is dependent only on the latter. Diffuse pleural thickening is characterised by the involvement of the visceral pleura and usually presents radiographically with blunting of the costophrenic angle. Unlike pleural plaques, which are localised lesions of the parietal pleura that do not affect respiratory mechanics, diffuse pleural thickening can cause significant restrictive impairment of ventilation, which can sometimes lead to chronic respiratory failure [24, 25]. Rounded atelectasis is a rare form of localised pleural thickening that presents as a round, masslike opacity with a characteristic curvilinear tail extending toward the hilum (i.e. comet tail sign) on chest computed tomography [26]. Although patients with rounded atelectasis are usually asymptomatic, they may become dyspnoeic when the volume of atelectatic lung is large enough to compromise lung function [27]. However, the main concern in patients with benign asbestos-related diseases is whether they represent independent risk factors for malignancy by adding risk to that of asbestos exposure alone. A first answer to this question has recently been provided by REID et al. [28], who found that pleural thickening and asbestosis appear to be associated with an increased risk of peritoneal but not pleural mesothelioma in a cohort of crocidolite exposed individuals. 


\section{Conclusion}

Asbestos is one of the most common avoidable hazards to the human lung. Although its association with a number of malignant and non-malignant lung diseases has been long ago established, novel aspects regarding the pathogenesis of these disorders are still coming to light. In this context, the multiplicative effect of smoking on lung cancer risk in asbestos-exposed individuals, the role of asbestos-exposure duration and level in the development of pleural plaques and asbestosis, and the absence of increased risk for malignant pleural mesothelioma among patients with asbestosassociated pleural thickening or asbestosis have recently been demonstrated.

\section{References}

1. Yang H, Testa JR, Carbone M. Mesothelioma epidemiology, carcinogenesis, and pathogenesis. Curr Treat Options Oncol 2008; 9: 147-157.

2. Lynch KM, Smith WA. Pulmonary asbestosis: carcinoma of the lung in asbestos-silicosis. Am J Cancer 1935; 24: 56.

3. Gloyne SR. Two cases of squamous carcinoma of the lung occurring in asbestosis. Tubercle 1935; 17: 5.

4. Doll R. Mortality from lung cancer in asbestos workers. $\mathrm{Br}$ J Ind Med 1955; 12: 81-86.

5. Selikoff IJ, Churg J, Hammond EC. Classics in oncology: asbestos exposure and neoplasia. CA Cancer J Clin 1984; 34: 48-56.

6. Raffn E, Lynge E, Korsgaard B. Incidence of lung cancer by histological type among asbestos cement workers in Denmark. $\mathrm{BrJ}$ Ind Med 1993; 50: 85-89.

7. Karjalainen A, Anttila S, Vanhala E, et al. Asbestos exposure and the risk of lung cancer in a general urban population. Scand J Work Environ Health 1994; 20: 243-250.

8. Stayner LT, Wegman DH. Smoking, occupation, and histopathology of lung cancer: a case-control study with the use of the Third National Cancer Survey. J Natl Cancer Inst 1983; 70: 421-426.

9. Frost G, Darnton A, Harding AH. The effect of smoking on the risk of lung cancer mortality for asbestos workers in Great Britain (1971-2005). Ann Occup Hyg 2011; 55: 239-247.

10. Elmes PC, Wade OL. Relationship between exposure to asbestos and pleural malignancy in Belfast. Ann N Y Acad Sci 1965; 132: 549-557.

11. Owen WG. Mesothelial tumors and exposure to asbestos dust. Ann N Y Acad Sci 1965; 132: 674-679.

12. Wagner JC. Epidemiology of diffuse mesothelial tumors: evidence of an association from studies in South Africa and the United Kingdom. Ann N Y Acad Sci 1965; 132 575-578

13. Butnor KJ, Sharma A, Sporn TA, et al. Malignant mesothelioma and occupational exposure to asbestos: an analysis of 1445 cases. Ann Occup Hyg 2002; 46: 150-153.

14. McDonald JC, Armstrong BG, Edwards CW, et al. Casereferent survey of young adults with mesothelioma: I. Lung fibre analyses. Ann Occup Hyg 2001; 45: 513-518.
15. Stanton MF, Layard M, Tegeris A, et al. Relation of particle dimension to carcinogenicity in amphibole asbestoses and other fibrous minerals. J Natl Cancer Inst 1981; 67: 965-975.

16. Hillerdal G. Mesothelioma: cases associated with nonoccupational and low dose exposures. Occup Environ Med 1999; 56: 505-513.

17. Hodgson JT, McElvenny DM, Darnton AJ, et al. The expected burden of mesothelioma mortality in Great Britain from 2002 to 2050. BrJ Cancer 2005; 92: 587-593.

18. McElvenny DM, Darnton AJ, Price MJ, et al. Mesothelioma mortality in Great Britain from 1968 to 2001. Occup Med (Lond) 2005; 55: 79-87.

19. Cooke WE. Pulmonary asbestosis. $\mathrm{Br}$ Med J 1927; 2 : 1024-1025.

20. McDonald S. Histology of pulmonary asbestosis. Br Med J 1927 ; 2: 1025-1026.

21. Pancoast HK, Miller TG, Landis HRM. A roentgenologic study of the effects of dust inhalation upon the lungs. Trans Assoc Am Physicians 1917; 32: 97.

22. Cauna D, Totten RS, Gross P. Asbestos bodies in human lungs at autopsy. JAMA 1965; 192: 371-373.

23. Paris C, Thierry $S$, Brochard $P$, et al. Pleural plaques and asbestosis: dose- and time-response relationships based on HRCT data. Eur Respir J 2009; 34: 72-79.

24. Jones RN, McLoud T, Rockoff SD. The radiographic pleural abnormalities in asbestos exposure: relationship to physiologic abnormalities. J Thorac Imaging 1988; 3: 57-66.

25. Schwartz DA, Galvin JR, Dayton CS, et al. Determinants of restrictive lung function in asbestos-induced pleural fibrosis. J Appl Physiol 1990; 68: 1932-1937.

26. Batra $\mathrm{P}$, Brown K, Hayashi $\mathrm{K}$, et al. Rounded atelectasis. J Thorac Imaging 1996; 11: 187-197.

27. Gaensler EA, Kaplan Al. Asbestos pleural effusion. Ann Intern Med 1971; 74: 178-191.

28. Reid A, de Klerk N, Ambrosini $G$, et al. The additional risk of malignant mesothelioma in former workers and residents of Wittenoom with benign pleural disease or asbestosis. Occup Environ Med 2005; 62: 665-669. 